Onkologe 2015 $21: 574-576$

DOI 10.1007/s00761-014-2855-3

Online publiziert: 1. Juli 2015

๑) Springer-Verlag Berlin Heidelberg 2015
M. Schwaiger ${ }^{1} \cdot$ K. Scheidhauer ${ }^{1} \cdot$ M. Bamberg ${ }^{2}$

${ }^{1}$ Nuklearmedizinische Klinik und Poliklinik, Klinikum rechts der Isar,

Technische Universität München

${ }^{2}$ Universitätsklinikum Tübingen

\title{
Multidisziplinärer Überblick über Diagnostik und Therapie des Schilddrüsenkarzinoms
}

Thema dieser Ausgabe der Zeitschrift Der Onkologe ist die Diagnostik und Therapie des Schilddrüsenkarzinoms. Die Beiträge sollen einen multidisziplinären Überblick aus Sicht der Chirurgie und der Pathologie, der Endokrinologie sowie der Nuklearmedizin vermitteln. Die Auswahl der Autoren entspricht der Entwicklung in der Onkologie, Tumorerkrankungen in spezialisierten interdisziplinären Teams zu behandeln, um rationale Diagnostik und Therapie den individuellen Bedürfnissen des einzelnen Patienten bestmöglich anzupassen. Das Schilddrüsenkarzinom ist schon immer ein Paradebeispiel für das erfolgreiche Zusammenspiel verschiedener Fachrichtungen, das in einem multimodalen Therapiekonzept zu sehr guten Ergebnissen geführt hat. Darüber hinaus finden regelmäßig nationale und internationale Kongresse statt, die regionale und fachspezifische Leitlinien vergleichend diskutieren und verbessern. Schilddrüsenzentren und multidisziplinäre Tumorboards bestimmen heute weltweit den Standard moderner individualisierter Therapie und Nachsorge dieser vergleichsweise seltenen Tumorerkrankung.

\section{》) Das Schilddrüsenkarzi- nom ist ein Paradebeispiel für das Zusammenspiel von Fachrichtungen}

Trotz einer sehr positiven Gesamtentwicklung ist die Diagnostik des Schilddrüsenkarzinoms in Deutschland durch überdurchschnittlich viele, unnötige Schild- drüsenoperationen charakterisiert. Laut einem Beitrag im Deutschen Ärzteblatt aus dem Jahr 2013 werden in Deutschland etwa 80.000 Schilddrüsenoperationen pro Jahr (davon rund 59.000 wegen einer Struma nodosa) durchgeführt, während z. B. in den USA die Zahl bei nur 75.000 Operationen liegt [1]. Die Malignitätsrate bei „diagnostischen Schilddrüsenoperationen" wegen eines Schilddrüsenknotens ist im europäischen Vergleich in Deutschland mit Abstand am niedrigsten und nicht allein durch eine höhere Knotenprävalenz zu erklären. Das Verhältnis der Operationen mit dem histologischen Ergebnis maligne vs. benigne Struma beträgt in Deutschland 1:15, in Italien 1:7, in England und in Skandinavien 1:5. Diese Zahlen weisen darauf hin, dass die diagnostischen Möglichkeiten zur Abklärung eines verdächtigen Schilddrüsenknotens nicht voll ausgenutzt werden und dadurch die präoperative Abschätzung des individuellen Malignitätsrisikos in Deutschland zu anderen Ergebnissen als in vergleichbaren Ländern führt. So wurde in dem untersuchten Kollektiv nur in $21 \%$ der Fälle mit uninodösen Schilddrüsenerkrankungen eine Feinnadelaspirationszytologie (FNAZ) durchgeführt.

Die genannten Zahlen können aber auch ein Hinweis darauf sein, dass die Indikationsstellung zur Schilddrüsenoperation in Deutschland oft auch durch weniger erfahrene oder weniger geschulte Ärzte gestellt wird. Entsprechend ist einzufordern, dass die diagnostischen Möglichkeiten durch interdisziplinäre Diagnose- und Behandlungswege - basierend auf den vorhandenen deutschen und inter- nationalen Leitlinien $\mathrm{zu}$ diesem Thema - besser ausgenutzt werden sollten, um die Zahl unnötiger Operation auch in Deutschland zu reduzieren.

Die hier publizierten Leitthemen sollen einen Beitrag dazu liefern, die ärztliche Herausforderung an eine optimierte Diagnostik aufzuzeigen und die Vorteile einer umfassenden, auch molekular-pathologischen Abklärung darzustellen. Es ist zu erwarten, dass die molekulare Diagnostik - auch von präoperativem FNAZ-Material - in Zukunft eine zunehmende Rolle nicht nur bei der Diagnostik eines Schilddrüsenkarzinoms, sondern auch bei der prognostischen Abschätzung eines pathologischen Befunds spielen wird. Dieser Aspekt wird in den Beiträgen von Prof. Schmid und Prof. Spitzweg dargestellt, denn das genetische Profil eines Tumors wird wesentlich den Verlauf und das Ansprechen auf zielgerichtete Therapien bestimmen.

Neben dem diagnostischen Vorgehen steht in den folgenden Leitthemenbeiträgen die derzeit intensive Diskussion einer leitliniengerechten Therapie des Schilddrüsenkarzinoms im Vordergrund. Technische Fortschritte und Standardisierung der chirurgischen Behandlung haben in den letzten Jahren zu niedrigen Komplikationsraten und nicht zuletzt auch zu sehr guten kosmetischen Ergebnissen geführt. Die überwiegend gute Langzeitprognose bei dieser Erkrankung ist Resultat der allgemein akzeptierten Kombination von chirurgischer und nuklearmedizinischer Behandlung.

Aufgrund der überproportional starken Zunahme niedriggradiger differen- 


\section{Hier steht eine Anzeige.}

Springer 
zierter Schilddrüsenkarzinome mit sehr guter Langzeitprognose, aber auch wegen der genannten guten Langzeiterfolge wird zunehmend der relative Beitrag der sog. primären Radiojodablation von verbliebenem Schilddrüsengewebe nach Operation in Zweifel gezogen. Breiter Konsens besteht bei sehr niedrigem Risiko (papilläres Karzinom $<1 \mathrm{~cm}$, Mikrokarzinom) über den Verzicht und bei sehr hohem Risiko (Karzinom $>4 \mathrm{~cm}$, Metastasen) über den Einsatz der Radiojodtherapie. Dazwischen liegt ein viel diskutierter Graubereich, der derzeit und sicher auch in Zukunft die interdisziplinären Tumorboards beschäftigen und weitere prospektive Studien erfordern wird. Solche Studien sind jedoch bei Erkrankungen mit guter Prognose eine große Herausforderung und daher kaum finanzierbar. Denn hierbei sind große Patientenzahlen und sehr lange Beobachtungszeiträume erforderlich, um statistisch relevante Aussagen treffen zu können.

\section{1) Bei disseminierten Metastasen bleibt die Hochdosis-Radiojodtherapie wichtigste Option}

Prof. Fürst und Prof. Luster legen in ihren Beiträgen die therapeutischen Möglichkeiten und das leitliniengerechte Zusammenspiel zwischen Chirurgie und $\mathrm{Nu}$ klearmedizin dar. Das betrifft nicht nur die Erstbehandlung des Schilddrüsenkarzinoms, sondern auch die Nachsorge und im Fall eines Progresses die erneute The rapie, um die Erkrankung möglichst lang unter Kontrolle zu halten. Die Nachsorge sollte risikoadaptiert angepasst werden und durch eine Kombination von In-vitro-Tumormarker-Untersuchung (hTG) und multimodaler Bildgebung gesteuert werden.

Bei Vorliegen von multiplen und disseminierten Metastasen bleibt die Hochdosis-Radiojodtherapie wichtigste Option, wobei viele Fragen über die genaue Aktivitätsmenge und Dosisbestimmung nicht vollständig beantwortet sind, wie Prof. Lassmann in seinem Beitrag differenziert diskutiert. Die Notwendigkeit einer regelhaften individuellen Dosimetrie steht jedoch aufgrund der hochspezifischen Jod- aufnahme der meisten differenzierten Schilddrüsenkarzinome und damit einem aus Sicht des Risikos ionisierender Strahlung sehr breiten Therapiefensters zurzeit nicht im Vordergrund.

Bei den relativ seltenen, meist fortgeschrittenen und metastasierten Schilddrüsenkarzinomen mit nachlassender oder fehlender Radiojodspeicherkapazität eröffnen sich in jüngster Zeit neue Therapieansätze, wie Prof. Spitzweg in ihrer Übersicht beleuchtet. Eine Vielzahl neuer Medikamente entweder zur Redifferenzierung der malignen Zellen oder zur Tumorkontrolle werden in naher Zukunft multizentrisch evaluiert oder zugelassen. Diese neuen Therapieoptionen erfordern neben der bereits erwähnten molekulardiagnostischen Klassifizierung eine enge interdisziplinäre Zusammenarbeit zwischen allen Therapeuten in der Auswahl der verfügbaren Therapieoptionen, um Erfolgsaussichten und Nebenwirkungsrisiken abzuwägen. Es besteht die begründete Hoffnung, dass auch die seltenen, aggressiveren und meist nichtjodspeichernden Formen des Schilddrüsenkarzinoms mit innovativen Substanzen in ihrem klinischen Verlauf verbessert werden können.

Wir danken allen Autoren herzlich für ihre Beiträge und hoffen im Namen aller Autoren, dass die geschilderten Fortschritte und neuen Therapieoptionen möglichst schnell allen Patienten mit Schilddrüsenkarzinom zugutekommen werden.

Für die Schriftleiter

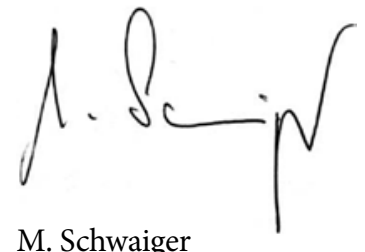

Für die Herausgeber
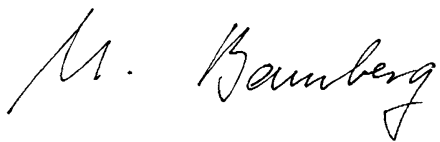

M. Bamberg

\section{Korrespondenzadresse

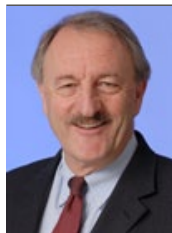 \\ Prof. Dr. M. Schwaiger \\ Nuklearmedizinische Klinik und Poliklinik, \\ Klinikum rechts der Isar, \\ Technische Universität \\ München \\ Ismaninger Straße 22, \\ 81675 München \\ markus.schwaiger@tum.de}

Interessenkonflikt. M. Schwaiger, K. Scheidhauer und M. Bamberg geben an, dass kein Interessenkonflikt besteht.

\section{Literatur}

1. Wienhold R et al (2013) Versorgung bei Schilddrüsenknoten: Eine retrospektive Analyse von Krankenkassendaten. Dtsch Arztebl Int 110(49): 827 834

2. Perros $P$ et al (2014) Guidelines for the management of thyroid cancer: Third edition. British Thyroid Association, July 2014. http://onlinelibrary.wiley com/doi/10.1111/cen.12515/pdf. Zugegriffen: 28. Mai 2015 